\title{
Cuerpos y planos geométricos usando realidad aumentada y computación afectiva
}

\author{
Ramón Zatarain-Cabada ${ }^{1}$, María Lucia Barrón-Estrada ${ }^{1}$, \\ María Blanca Ibañez-Espiga ${ }^{2}$, Aldo Uriarte-Portillo ${ }^{1}$ \\ ${ }^{1}$ Instituto Tecnológico de Culiacán, Culiacán, Sinaloa, México \\ ${ }^{2}$ Universidad Carlos III de Madrid, Departamento de Ingeniería Telemática, España \\ \{rzatarain, lbarron, auriarte\}eitculiacan.edu.mx, \\ mbibanezlit.uc3m.es
}

\begin{abstract}
Resumen. La adquisición de competencias en Ciencia, Tecnología, Ingeniería y Matemáticas será esencial para que las nuevas generaciones puedan enfrentar el competitivo mundo donde les tocará desenvolverse. $\mathrm{Si}$ bien nuestros estudiantes están rodeados de estímulos tecnológicos que los apasionan y a veces distraen, los sistemas de aprendizaje no están aprovechando estas tecnologías para atraer la atención de los estudiantes, facilitarles la comprensión de los temas abstractos propios de estas asignaturas. El trabajo que aquí se presenta pretende contribuir a la enseñanza de los temas "Cuerpos y Planos Geométricos" facilitando el aprendizaje gracias a las posibilidades de visualización tridimensional e interacción en tiempo real que ofrece la tecnología de realidad aumentada e incrementando el nivel de motivación utilizando técnicas de computación afectiva. A partir de los requerimientos educativos realizados por 7 profesores de diferentes escuelas secundarias, se diseñaron actividades en un entorno de realidad aumentada para facilitar el aprendizaje de "Cuerpos y Planos Geométricos". Las actividades están integradas en un entorno educativo utilizando técnicas de computación afectiva.
\end{abstract}

Palabras clave: realidad aumentada, inteligencia artificial, computación afectiva, reconocimiento de emociones, aprendizaje móvil.

\section{Geometric Shapes and Planes Using Augmented Reality and Affective Computing}

\begin{abstract}
The acquisition of competences in Science, Technology, Engineering and Mathematics will be essential so that the new generations can face the competitive world where they will have to progress. Although our students are surrounded by technological stimuli that are passionate and sometimes distracting, the learning systems are not taking advantage of these technologies to attract the attention of the students, to facilitate their understanding of the abstract topics of these subjects. The work presented here aims to contribute to the teaching of the topics "Geometric Shapes and Planes" by facilitating learning thanks to the possibilities of three-
\end{abstract}


dimensional visualization and real-time interaction offered by augmented reality technology and by increasing the level of motivation using techniques of Affective Computing. Based on the educational requirements made by 7 teachers from different middle and high schools, activities were designed in an augmented reality environment to facilitate the learning of "Geometric Shapes and Planes". The activities are integrated into an educational environment using affective computing techniques.

Keywords: augmented reality, artificial intelligence, affective computing, affective recognition, mobile learning.

\section{Introducción}

La Agenda 2030 para el Desarrollo Sostenible [1] propone un plan de acción para enfocar de manera integrada las causas que permitan la superación de la pobreza, para orientar el desarrollo hacia la sostenibilidad y el establecimiento de una calidad de vida digna para todos. Los aportes de las ciencias, la tecnología, la innovación son indispensables para que se avance en las direcciones deseadas [2] La educación científica, en la educación obligatoria, debe asegurar a todos sus estudiantes aprendizajes de calidad. Sin embargo, el escenario de la región latinoamericana muestra que la educación no solo no brinda estos aprendizajes, sino que la manera como se presenta el conocimiento científico tiende a que los jóvenes pierdan el interés por aprender ciencias, y no se despierten vocaciones científicas [2].

En los últimos años, los investigadores están explorando las posibilidades educativas de nuevas tecnologías emergentes como la realidad aumentada, los mundos virtuales guiados por métodos como la computación afectiva, y la gamificación para diseñar nuevos entornos educativos que faciliten y mejoren el aprendizaje. Las nuevas tecnologías aplicadas a la educación están empezando a producir aplicaciones con nuevos métodos como es el caso de la realidad aumentada [3,4] o los sistemas reconocedores de emociones [5,6] cuya aplicación pueden facilitar el aprendizaje. La realidad aumentada (RA) es la tecnología que permite aumentar la percepción del mundo real con información digital superpuesta en el mundo real. La información puede consistir en texto, imágenes, audio, video. El usuario tiene la posibilidad de interactuar en tiempo real con la información digital, esto incluye la observación tridimensional de la información digital. Esta tecnología requiere de elementos visuales que permitan mostrar la información relevante en cada momento. Estos elementos son imágenes o puntos geolocalizados.

En 2016 hubo un incremento del 5\% del uso de dispositivos móviles respecto a 2015 [7]. El tiempo que los usuarios dedican a usar un dispositivo móvil cada vez es mayor. Hoy en día un gran número de personas con diferente edad, género, condición social, nivel educativo usan algún dispositivo móvil desde cualquier lugar, a cualquier hora, y con ello pueden realizar alguna actividad recreativa, capturar algún momento importante, o incluso realizar alguna tarea de su área de trabajo. Estas acciones generan una gran cantidad de información de diversos tipos que puede ser utilizada 
con fines de investigación, tal es el caso del reconocimiento de emociones en tiempo real desde un ambiente natural.

Por otra parte, la enseñanza de las Matemáticas, particularmente el área de Geometría, causa dificultades a los estudiantes debido al esfuerzo de abstracción que deben realizar para imaginar cuerpos en el espacio tridimensional cuando las explicaciones se realizan en pizarrón, un espacio bidimensional. La posibilidad que la tecnología de RA brinda de poder interactuar en tiempo real con elementos digitales tridimensionales, brinda potencialmente dos posibilidades. Por un lado facilitar la comprensión y ejercitación de los temas de geometría a tratar. Por otro lado, ayudar a aumentar los niveles de interés y concentración de los estudiantes involucrándolos en el aprendizaje activo que será soportado por el entorno educativo que aquí se presenta.

La principal contribución de este trabajo es el diseño y la implementación de un entorno de aprendizaje que permite integrar la tecnología de realidad aumentada con un sistema reconocedor de emociones. El diseño se realiza a partir del análisis de las necesidades educativas en un entorno real a partir de las opiniones de profesores que dictan el tema de Geometría en educación Secundaria en México El ambiente complementa un entorno real superponiendo información digital a través de modelos en 3D, logrando obtener la atención de los estudiantes al visualizar los elementos geométricos desde la perspectiva elegida, fomenta el aprendizaje activo y por último, adapta las actividades a realizar en función de los estados de ánimo del estudiante.

Este artículo está estructurado en el siguiente orden: en la Sección 2 presentamos los trabajos relacionados en las áreas de computación afectiva y en realidad aumentada orientada a la educación; en la Sección 3 se presenta un análisis de las entrevistas realizadas con los profesores; en la Sección 4 se plantea la estructura del entorno de aprendizaje; en la Sección 5 se describen las evaluaciones de la herramienta de RA realizadas con profesores, y para finalizar en la sección 6 se presentan las conclusiones y trabajos futuros.

\section{Trabajos relacionados}

En esta sección se describen algunos trabajos de investigación relacionados en el campo de la computación afectiva y de realidad aumentada en la educación. Estos trabajos guardan similitud con este trabajo de investigación y fueron considerados como punto de partida para el desarrollo de este proyecto.

En el reconocedor facial presentado por Khandait et al. [8] detecta una imagen y realiza el reconocimiento facial con base en distancias entre rasgos faciales. Este sistema utiliza como clasificador a las redes neuronales artificiales con retro propagación y reconoce las emociones básicas de sorprendido, neutral, triste, disgustado, feliz y enojado. Para la extracción de características implementa SUSAN para la detección de bordes en cejas, ojos, boca y nariz, alcanzando una precisión de efectividad de $95.26 \%$.

El proyecto propuesto por Patwardhan et al. [9] es un sistema desarrollado que utiliza múltiples modalidades, como cara, mano, cabeza y habla para reconocer el 
estado afectivo de un individuo, por ello utilizan un dispositivo que permite detectar color y profundidad como el Microsoft Kinect; con este hardware pueden realizar la extracción de características del rostro, y del movimiento de articulaciones. El autor señala que se centra en las emociones básicas. Utiliza como clasificador una Maquina de Vector Soporte (SVM por sus siglas en inglés) para el reconocimiento de emociones en cada modalidad. Patwardhan y sus colegas, obtuvieron una precisión de reconocimiento de emociones en rostro del $84 \%$ en las emociones de felicidad, $77 \%$ en la emoción de enojo y $71 \%$ en tristeza, en tanto modo multimodal fue del $88 \%$, $83 \%$ y $77 \%$ respectivamente.

El proyecto de Wang et al. [10] también implementa como clasificador una SVM por su buen rendimiento para el reconocimiento de patrones, específicamente en el reconocimiento de emociones a través de la voz. El clasificador fue entrenado con base de datos con voz etiquetada del idioma alemán, chino, y emociones de la antigua china. El modelo de datos del proyecto implementa los parámetros de Fourier (FP por sus siglas en inglés) y el coeficiente cepstral de frecuencias Mel (MFCC). Los resultados obtenidos tienen una precisión promedio del $87.5 \%$.

En el marco del uso de la realidad aumentada en el campo educativo, los estudios iniciales han estado dedicados principalmente a estudiar factores como la usabilidad y la motivación en prototipos educativos. Aplicaciones basadas en marcadores se han utilizado para ayudar a los estudiantes a mejorar sus habilidades espaciales. Así, Liao et al. [11] desarrollaron una herramienta en Unity, Vuforia y JavaScript como motor de scripts que facilita a los estudiantes resolver el problema de un cubo de Rubik. Ellos implementan la RA para mostrar pistas en el proceso de solución del cubo de Rubik además de aprender conceptos elementales de volumen y superficie de cuerpos geométricos. El proyecto está en fase de pruebas con el fin de evaluar la eficiencia del aprendizaje esperado en los estudiantes.

El proyecto ARMatika desarrollador por Young et al. [12] es un juego que implementa un conjunto de elementos enfocados a mejorar el aprendizaje del estudiante, utilizando efectos visuales, técnica de puntajes, reglas de juego, movimiento de personajes y donde se tiene un numero de aciertos y errores permitidos. El porcentaje de preferencia de sus estudiantes para aprender es del $85.3 \%$.

En el estudio realizado por del Cerro et al. [13] abordan temas de figuras geométricas adaptadas al plan de estudio de tercer grado de secundaria, con herramientas como Junaio y Sketchup. Las actividades educativas consistieron en ejercicios prácticos en donde usando marcadores QR convencionales obtenían diferentes vistas de la figura solicitada. El $82.6 \%$ quedo satisfecho con el uso de la herramienta diseñada y el $78.26 \%$ de los participantes afirman haber comprendido con mayor claridad el tema visto en clase al usar la herramienta.

\section{Análisis de entrevistas a profesores}

El primer paso antes de realizar el diseño del entorno de aprendizaje fue entrevistar a un grupo de 7 profesores que imparten la materia de matemáticas en el segundo y 
tercer grado de escuelas mexicanas. De acuerdo a un análisis que llevamos a cabo de encuestas abiertas realizadas a los profesores podemos establecer que:

(1) 6 de 7 docentes afirman que la enseñanza de la Geometría es un reto debido a la necesidad de utilizar un instrumento como el pizarrón para explicar conceptos tridimensionales.

(2) 5 de 7 docentes indicaron que los estudiantes manifiestan poco interés en el estudio de temas ligados a las matemáticas.

(3) 6 de 7 docentes indican que a sus estudiantes les atrae más el uso de un dispositivo móvil que hacer actividades en su pupitre con papel y lápiz.

(4) 4 de 7 docentes indican que los estudiantes por timidez o temor a equivocarse participan poco en las actividades en el aula.

(5) 4 de 7 docentes indican que el nivel de los estudiantes en sus clases es variable.

Con base a lo anterior podemos establecer las siguientes implicaciones que corresponden a cada una de las respuestas de los profesores:

La respuesta 1 implica que la RA puede ser el uso de la tecnología de realidad aumentada para la realización de actividades educativas en el campo de la Geometría porque la realidad aumentada le permite la visualización en tiempo real de fenómenos que ocurren en espacio 3D, logrando disminuir la dificultad para comprender, visualizar, imaginar y asociar elementos geométricos.

La respuesta 2 implica que la RA puede ser útil, ya que estudios como [11,12 13] indican que la RA fomenta la motivación de los estudiantes, llamando su atención, comprometiéndolos a realizar la actividad y con ello facilitar su aprendizaje.

La respuesta 3 implica que la RA puede ser útil puesto que requiere de uso de dispositivos móviles con los cuales los jóvenes estudiantes están familiarizados.

La respuesta 4 implica que la RA tendría un efecto positivo porque permite la interacción individual, la retroalimentación en el mismo momento con evaluación formativa, aumentando la actividad del estudiante en el tema.

Respuesta 5 implica que la RA combinada con la computación afectiva puede tener impacto positivo ya que permite colocar elementos de andamiaje educativo que se ajusten al perfil general y actual de cada estudiante.

\section{Estructura del entorno de aprendizaje}

En esta sección se presenta la arquitectura de la aplicación y los marcadores diseñados para el ambiente de aprendizaje.

\subsection{Arquitectura}

ARGeo es una aplicación móvil desarrollada en Unity implementando el sistema de reconocimiento de marcadores Vuforia y Scripts programados en C\#, la cual fue diseñada para los sistemas operativos Android e IOS. ARGeo le muestra al estudiante un conjunto de ejercicios de área, volumen y cortes aplicables a cuerpos geométricos; mientras el estudiante interactúa con la aplicación, ésta recolecta una imagen y clasifica la emoción detectada mostrándola en pantalla. El componente principal del 
dispositivo móvil que tiene función en este proyecto es la cámara. En la Figura 1 se presenta la arquitectura que fue diseñada para esta aplicación, la cual está formada por los componentes Vista, Control y Reconocedor.

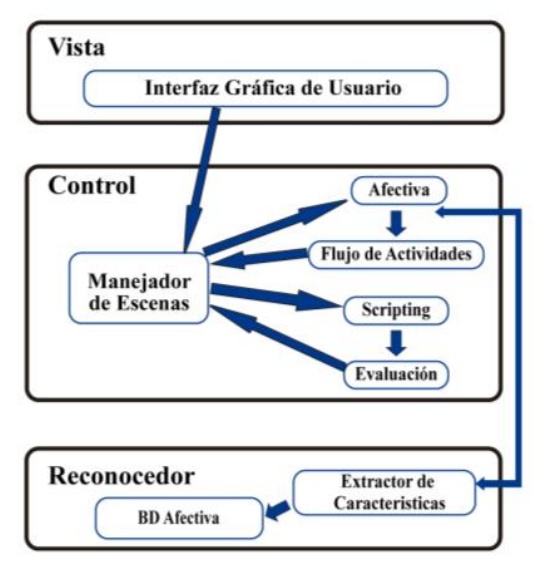

Fig. 1. Arquitectura de ARGeo.

La capa Reconocedor fue implementada mediante una red neuronal convolucionada $(\mathrm{CNN})$ utilizada para identificar las emociones a través de imágenes tomadas a los estudiantes [14]. La CNN contiene tres capas convolucionales, tres capas de tipo "max-pooling" y tres redes neuronales con conexiones intermedias de tipo "dropout" (ver Figura 2).

La CNN fue entrenada usando tres diferentes bases de datos afectivas: una base de datos con emociones actuadas y dos bases de datos de emociones espontáneas creadas especialmente en el Laboratorio de Computación Móvil y Afectiva del Instituto Tecnológico de Culiacán, con un contenido orientado en las emociones centradas en el aprendizaje (emocionado, comprometido, aburrido o frustrado). La red neuronal se encarga de extraer las características del rostro del estudiante y de acuerdo a ellas reconocer la emoción del mismo. La emoción es enviada al módulo Afectiva para después ser utilizada por el módulo de flujo de actividades.

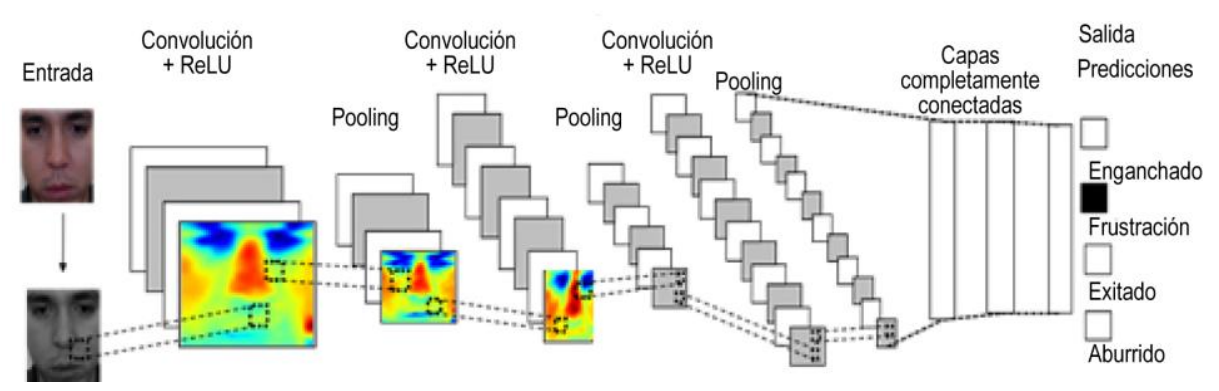

Fig. 2. Arquitectura de una CNN para Reconocer Emociones. 
En la capa Control se efectúa el manejo de escenas mediante la superposición de la información digital sobre el entorno real; esta información digital puede ser audio, video, imagen, elementos 3D y animaciones. El módulo Afectiva quien recibe del reconocedor la emoción actual del estudiante, guía al módulo de Flujo de Actividades para mostrar al estudiante las posibles actividades que él requiera. El estudiante ejecuta una actividad mediante un script, realiza la interacción y el módulo de evaluación recibe cómo fue la actuación del estudiante. El módulo de evaluación le indica al estudiante cómo lo hizo y esto genera un posible cambio en las emociones del estudiante. Este estado de ánimo es recogido por el módulo de computación afectiva.

Para poder realizar realidad aumentada se requiere de diversos elementos: una cámara que capte una imagen del espacio real, un programa que interprete y reconozca la imagen captada y que pueda hacer la fusión con el entorno real, y una pantalla donde se pueda observar la combinación de la realidad con elementos virtuales y los marcadores [14]. Para conseguirlo se utiliza el entorno Unity 2017 [15] con Vuforia 7.2 [16]. El dispositivo desde el cual se utiliza la aplicación debe contar con una cámara. Para que se efectúe el reconocimiento de un marcador es necesario colocarlo en el campo de visión de la cámara, de tal manera que la cámara pueda detectar el patrón adecuadamente. En este proceso juega un rol importante el motor de realidad aumentada (Vuforia), el cual al reconocer el marcador superpone los elementos digitales (e.g. imágenes, objetos 3D o animaciones) sobre el objeto, para poder asignar algún proceso o realizar alguna operación en específico y así tener el objeto ya aumentado [17]. En este proyecto se ha utilizado el uso de comportamientos de colisiones y de movimiento de objetos.

Por último, la capa Vista contiene la interfaz principal que está formado por un menú que permite elegir una de las tres actividades educativas (ejercicios) diseñadas. En la Figura 3 podemos apreciar su menú principal y una vista previa de cada ejercicio. Se implementaron tres ejercicios:

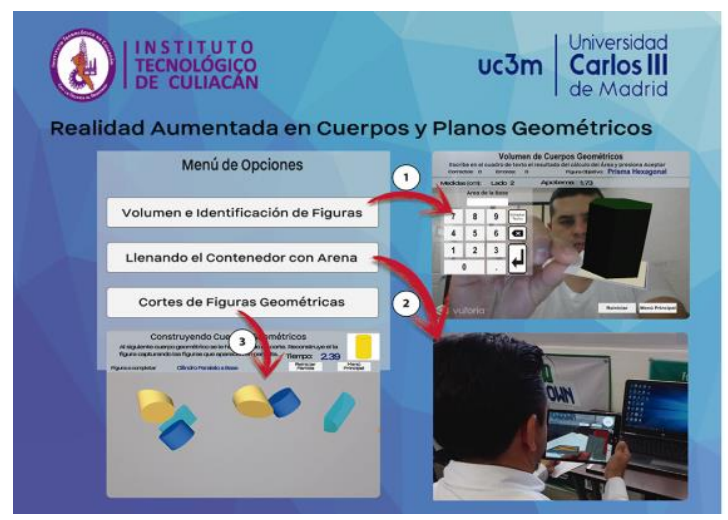

Fig. 3. Interfaz principal del Ambiente de Realidad Aumentada.

El primer ejercicio consiste de cálculo de área y volumen de prismas regulares donde el estudiante debe reconocer el marcador que hace referencia al cuerpo 
geométrico que la aplicación le está solicitando. Si el estudiante acierta al seleccionar el marcador se plasman en pantalla la información necesaria para realizar los cálculos de área de la base y de volumen; en tanto en caso contrario se despliega un mensaje indicando al usuario que se ha equivocado.

El segundo ejercicio consiste en ver en tiempo real como se van acumulando volúmenes de prismas regulares. Para ello, el usuario debe colocar el marcador que hace visible el prisma regular al cual se pretende obtener textura de él. En la Figura 4 se puede apreciar este procedimiento. Una vez visible, se requiere elegir la medida correspondiente para llenar de textura el objeto. Con un marcador denominado cubo se realizan las colisiones a los prismas objetivo y en pantalla se va visualizando el llenado de textura de un prisma a otro, mientras se aprecia el incremento del volumen de la textura del prisma regular y en pantalla se refleja en centímetros cúbicos el tamaño textura que se ha obtenido hasta la última colisión. El ejercicio culmina al completar el cien por ciento de la textura en el prisma contenedor.

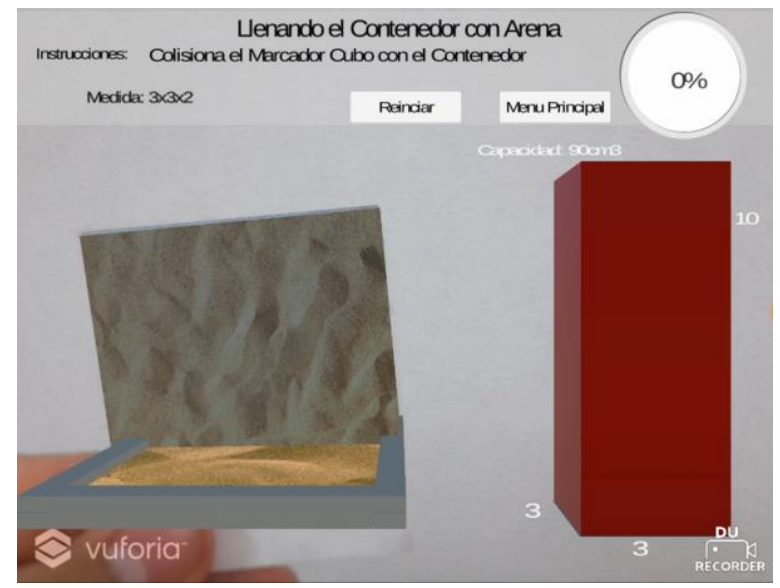

Fig. 4. Marcador obteniendo textura del objeto al colisionar con otro objeto.

El tercer ejercicio consiste en identificar correctamente las secciones de corte a cuerpos geométricos. Este ejercicio aplica la lluvia de objetos con el afán de que el estudiante vea desde diferentes ángulos y posturas el corte que se le pide y con ello reforzar su aprendizaje en el tema.

\section{Evaluación con profesores}

En esta sección abordaremos la evaluación y retroalimentación que los profesores de la asignatura de Matemáticas llevaron a cabo. A cada profesor se le explicó el objetivo de evaluar la herramienta de aprendizaje aplicando la tecnología de realidad aumentada. Posteriormente los profesores probaron el funcionamiento de la herramienta de RA directamente en el dispositivo móvil. El proceso de evaluación se clasificó en dos fases: la primera fase consiste en evaluar la parte pedagógica de la 
aplicación, donde los profesores nos expresaron que el ambiente de aprendizaje es factible aplicarlo a segundo y tercer grado. Además, nos argumentaron que los alumnos debían contar con algunos conocimientos previos como conceptos de área, volumen e identificación de cuerpos geométricos.

En la parte pedagógica los profesores hicieron hincapié en el aspecto motivacional del estudiante, donde los profesores consideraron que el ambiente de aprendizaje influye en fomentar la motivación del estudiante por aprender temas de geometría. También se consideraron algunos aspectos como, el tiempo que toma comprender el funcionamiento de cada actividad, las repeticiones de cada ejercicio para que el alumno domine su funcionamiento y la reflexión del aprendizaje adquirido. En la segunda fase los profesores evaluaron la usabilidad de la aplicación, expresando, si la herramienta les resultó innovadora y fácil de usar por parte de los alumnos, concluyendo que les sería muy útil y que les gustaría implementarlo en sus prácticas docentes. Los profesores interactuaron con la aplicación y nos expresaron algunas observaciones que nos permitieron mejorar el ambiente de aprendizaje.

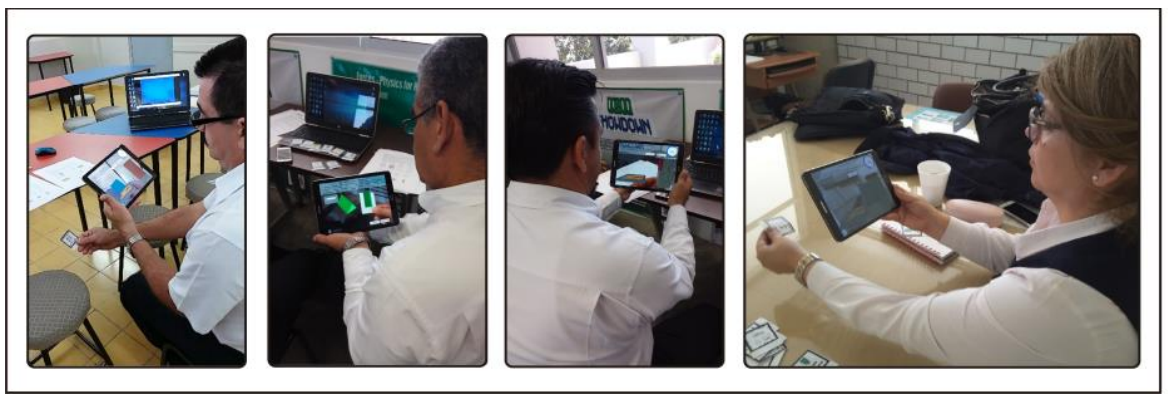

Fig. 5. Evaluación del ambiente por los Profesores.

Tabla 1. Comentarios relevantes por parte de los Profesores.

\begin{tabular}{|c|c|}
\hline Docente & Comentario \\
\hline $\begin{array}{l}\text { Profesor } \\
1\end{array}$ & $\begin{array}{l}\text { Los adolescentes están en la era de la tecnología. Para ellos todo lo que es } \\
\text { tecnología y algo diferente en áreas lúdicas es muy motivador. La aplicación } \\
\text { ayudaría a reforzar y mejorar el conocimiento del tema, puesto que el alumno es } \\
\text { capaz de ver la geometría desde otra perspectiva. }\end{array}$ \\
\hline $\begin{array}{l}\text { Profesor } \\
2\end{array}$ & $\begin{array}{l}\text { Respecto a los temas que se abordaron la aplicación es de mucha utilidad, fomenta } \\
\text { la motivación, permite que el alumno tenga un aprendizaje mayor y de una forma } \\
\text { sencilla de comprender y va de acuerdo a la época en que se está viviendo. }\end{array}$ \\
\hline $\begin{array}{l}\text { Profesor } \\
3\end{array}$ & $\begin{array}{l}\text { La aplicación de RA les ayuda mucho a visualizar en que están trabajando, } \\
\text { mostrarles de manera más clara lo que ellos pueden ver, manipular, y conocer } \\
\text { fácilmente en comparación a si lo observas a través de una formula en el pizarrón, } \\
\text { donde el conocimiento no queda tan abstracto y ni tan claro. }\end{array}$ \\
\hline $\begin{array}{l}\text { Profesor } \\
4\end{array}$ & $\begin{array}{l}\text { Sería una herramienta más para mí y tendría un valor agregado para mi materia. } \\
\text { Considero que motivaría a mis alumnos, aprenderían más, no los aburriríamos con } \\
\text { lo mismo, el libro, la libreta, y de este modo los motivaría más hacia la asignatura } \\
\text { de matemáticas que por lo regular no les agrada. }\end{array}$ \\
\hline $\begin{array}{l}\text { Profesor } \\
5\end{array}$ & $\begin{array}{l}\text { La verdad esta herramienta ayudaría muchísimo y atacaríamos el problema de } \\
\text { identificación de cuerpos, donde no saben identificar el nombre de cada cuerpo. Al } \\
\text { momento de ver los cuerpos y manipularlos les queda más claro, ya que pueden } \\
\text { observar la base, manipular el cuerpo de tal forma que les queda más claro. La } \\
\text { conclusión a la que pueden llegar es que al momento de estar llenando un } \\
\text { recipiente de manera virtual, la fórmula de volumen le queda más claro. }\end{array}$ \\
\hline
\end{tabular}


En la Figura 5 podemos observar a algunos de los profesores interactuando con la herramienta y en Tabla 1 podemos apreciar los comentarios más relevantes que los docentes hicieron respecto al entorno de aprendizaje.

\section{Conclusiones y trabajos futuros}

El sistema desarrollado es capaz de realizar el reconocimiento de emociones utilizando la información de la cámara de un dispositivo móvil a través de la interacción con los ejercicios. Cabe destacar que el reconocedor está diseñado para que interactúe con ARGeo o alguna otra aplicación que requiera de reconocer emociones. El sistema reconocedor solo está disponible a través de un servicio web que recibe como parámetro la imagen en formato de datos binarios que la conforman y da como resultado una clase, que equivale a la emoción que le corresponde.

La primera evaluación que se llevó a cabo de la herramienta de RA y computación afectiva aquí mostrada fue con profesores que imparten la materia de matemáticas a nivel secundaria. De esta evaluación se concluyó que los profesores creen que los estudiantes podrían sentirse más motivados en su aprendizaje y que los temas abordados sobre cuerpos geométricos tridimensionales pueden ser más simples de comprender. Por otra parte, los profesores hicieron algunas observaciones sobre detalles del software que fueron tomadas en cuenta y en su caso corregidas.

Dentro del marco de los trabajos futuros se encuentran a corto plazo el iniciar pruebas con estudiantes de las 7 instituciones educativas para así analizar el impacto de nuestra herramienta con los estudiantes, tanto en aspectos de motivación y usabilidad como en ganancia de aprendizaje. También queremos personalizar el aprendizaje de tal forma que se tomen en cuenta los resultados de los ejercicios más el estado afectivo del estudiante para determinar rutas de aprendizaje para los estudiantes.

Agradecimientos. Agradecemos a las instituciones educativas Colegios Sinaloa, A.C., Colegio Chapultepec, Escuela Secundaria Técnica \# 19, Escuela Secundaria 24 de agosto y a la Escuela Secundaria Técnica \# 92 ubicadas en la ciudad de Culiacán, Sinaloa, así como a la Escuela Secundaria Técnica \# 38 ubicada en Guasave, Sinaloa, que nos han apoyado desde un inicio en este proyecto para poder llevar a cabo las evaluaciones de nuestro software. Asimismo, agradecemos a los docentes del área de geometría que con su experiencia contribuyeron al diseño de los ejercicios contemplados en este trabajo.

\section{Referencias}

1. United Nations: Transforming our world: the 2030 Agenda for Sustainable Development. General Assembly Resolution 70/1, pp. 1-40. Recuperado a partir de http://unctad.org/meetings/es/SessionalDocuments/ares70dl_es.pdf (2015)

2. Macedo, B.: Educación científica. Organización de las Naciones Unidas para la Educación, la Ciencia y la Cultura-UNESCO (2016)

3. Bower, M., Howe, C., McCredie, N., Robinson, A., Grover, D.: Augmented Reality in 
education-cases, places and potentials. Educational Media International, 51(1), pp. 115 (2014)

4. Radu, I.: Augmented reality in education: a meta-review and cross-media analysis. Personal and Ubiquitous Computing, 18(6), pp. 1533-1543 (2014)

5. Corneanu, C. A., Simón, M. O., Cohn, J. F., Guerrero, S. E.: Survey on rgb, 3d, thermal, and multimodal approaches for facial expression recognition: History, trends, and affectrelated applications. IEEE transactions on pattern analysis and machine intelligence, 38(8), pp. 1548-1568 (2016)

6. Zatarain-Cabada, R., Barrón-Estrada, M. L., Hernández, F. G., Alor-Hernandez, G.: Evaluando afecto en un entorno de aprendizaje para Java. Research in Computing Science, 111, pp. 123-133 (2016)

7. México edition, Deloitte Global Mobile Consumer Survey, May - Jun 2015, May - Jun 2016 (2016)

8. Khandait, S. P., Thool, R. C., Khandait, P. D.: Automatic facial feature extraction and expression recognition based on neural network. arXiv, pp.1204-2073 (2012)

9. Patwardhan, A., Knapp, G.: Multimodal Affect Recognition using Kinect. arXiv preprint arXiv: 1607-02652 (2016)

10. Wang, K., An, N., Li, B. N., Zhang, Y., Li, L.: Speech emotion recognition using fourier parameters. IEEE Transactions on affective computing, 6(1), pp 69-75 (2015)

11. Liao, Y. T., Yu, C. H.Wu, C. C.: Learning geometry with augmented reality to enhance spatial ability. In: Learning and Teaching in Computing and Engineering (LaTiCE), 2015 International Conference on IEEE, pp. 221-222 (2015)

12. Young, J. C., Kristanda, M. B., Hansun, S.: ARmatika: 3D game for arithmetic learning with Augmented Reality technology. In: Informatics and Computing (ICIC), International Conference on IEEE, pp. 355-360 (2016)

13. del Cerro Velázquez, F., Méndez, G. M.: Realidad Aumentada como herramienta de mejora de la inteligencia espacial en estudiantes de educación secundaria. Revista de Educación a Distancia, pp 54 (2017)

14. Gonzalez-Hernandez, F., Zatarain-Cabada, R., Barrón-Estrada, M.L., Rodríguez-Rangel, H.: Recognition of learning-, entered emotions using a convolutional neural network. In: Mexican International Conference on Artificial Intelligence. Springer, Cham (2017).

15. Unity Homepage (2018)

16. Vuforia Homepage, Developer portal (2018)

17. Patil, S., Prabhu, C., Neogi, O., Joshi, A. R., Katre, N.: E-learning system using Augmented Reality. In: Computing Communication Control and automation (ICCUBEA), 2016 International Conference on IEEE, pp. 1-5 (2016) 